\title{
VISUAL INFORMATION PROCESSING BY INTRACEREBRAL RETINAL TRANSPLANTS IN RATS
}

\author{
R. D. LUND ${ }^{1}$ and P. J. COFFEY ${ }^{2}$ \\ Cambridge and Sheffield
}

\begin{abstract}
SUMMARY
We have developed a simple system involving the implantation of retinae over the midbrain of rodents to examine whether, in a clearly defined system such as the primary optic pathway, it is possible to re-create circuits lost as a result of injury or developmental disorder. For much of the work, immature rat hosts have been used, in part to maximise optimal conditions and to provide a baseline for similar transplants in adults. In this review we summarise the sequence of studies that has led us to the conclusion that transplanted retinae are capable not only of differentiating and responding to light but also of relaying luminance information to visual centres of the host brain where appropriate behavioural responses are elaborated.
\end{abstract}

Work over the past 15 years has shown that it is possible to transplant embryonic neural tissue to the central nervous system of mammals. Such tissue differentiates relatively normally and integrates with the host systems sufficiently well to achieve some degree of recovery of behaviours lost as a result of induced or genetically controlled degeneration. While major attention has been given to those transplants that promote recovery largely using cells whose main role appears to be to provide a missing chemical in a target area, ${ }^{1,2}$ transplants may also function by recovering damaged neural circuitry. ${ }^{3.4}$ To achieve this, a much more complex set of requirements must be met. The transplant must be able to self-differentiate and form quite precise afferent and efferent connections which will permit it to analyse input signals and redistribute them in a suitably encoded fashion to regions of the host brain where they can mediate normal responses to defined stimuli. One important step in examining exactly how good transplants may be is to study as simple a system as possible, where the transplant function can be measured unambiguously,

From 'Department of Anatomy, University of Cambridge, Cambridge; ${ }^{2}$ Department of Psychology, University of Sheffield, Sheffield, UK.

Correspondence to: R. D. Lund, Department of Anatomy, University of Cambridge, Downing Street, Cambridge CB2 3DY, UK. background responses from residual or alternative pathways can be eliminated, and the pathway is a one-way system in which reciprocal interactions between transplant and host are not an essential part of the response.

One situation that answers these requirements is achieved by transplanting the retina over the midbrain of newborn and adult rats. Such retinae survive, differentiate, and connect with visual centres in the host rat brain. ${ }^{5}$ These connections are capable of transmitting light-driven information to the host brain in a manner that can be used to direct host behaviour patterns. In this review we will outline the principal findings.

\section{RETINAL DIFFERENTIATION}

Retinae are taken for implantation at between 12 and 15 days of gestation. They are removed from the host eye, and dissected free from the investing layers including the pigment epithelium in a dish of ice-cold tissue culture medium. At the time of explantation, each retina appears as an undifferentiated layer of cells, most of which have yet to undergo their terminal mitosis. After implantation into the host brain, they show a relatively normal pattern of differentiation: ${ }^{6}$ the usual retinal layers can be recognised and when labelled specifically, the cells show features characteristic of their normal in vivo appearance. ${ }^{7}$ Outer segments form on schedule, although they fail to line up in the orderly manner seen in normal eyes. The grafts placed in neonatal hosts can frequently adopt the configuration of a normal retina, although there is a tendency, when damaged, for them to show areas of rosetting. This is seen at its most extreme when the retinae are dissociated and reaggregated prior to transplantation, ${ }^{8}$ but even under these conditions the laminar patterns are still evident. Transplants placed in neonatal hosts can survive for as long as 2 years: allografts rarely show signs of rejection while xenografts show evidence of rejection in about a third of cases.9-11

It should be noted that in dissecting out the retinae the pigment epithelium is removed. This might be expected to cause loss of receptor cells, since in mutant strains a deficit

Eye (1994) 8, 263-268 C 1994 Royal College of Ophthalmologists 
targeted to the pigment epithelium causes relatively rapid receptor cell loss occurring over the first postnatal month and a slower decline over the succeeding months. ${ }^{12}$ For the grafts, it appears that microglial cells assume the phagocytic role of the pigment epithelium: ${ }^{13}$ however, although receptors do persist for more than 6 months post-transplantation, there is subsequently a slow loss with time, so that by 1 year it is extremely difficult to see any evidence of cells belonging to the outer nuclear layer. As in the case of retinal degeneration mutants, despite this loss of receptors there is still some level of residual function, in terms of both pupilloconstrictor response and conditioned suppression response (see below for details of the testing paradigm).

In general, grafts placed in adult hosts rather than neonates fare much less well, showing much more evidence of rosetting with less of the retina organised in an ordered laminar array. This presumably reflects the fact that the adult brain is much less permissive of maturational processes in general, and perhaps that reactive responses in the adult brain are more severe than those in immature brains.

\section{CONNECTIVITY}

Within a day of transplantation, retinae implanted in neonatal rat emit axons and over succeeding days these show a highly specific and directed outgrowth to a number of subcortical visual centres. ${ }^{14}$ They do not innervate non-visual nuclei, except possibly in the immediate vicinity of the graft. In a majority of cases, the superior colliculus, pretectal region and accessory optic nuclei are all innervated quite heavily by transplant-derived axons. ${ }^{8.15}$ The dorsal lateral geniculate nucleus generally receives a sparse innervation which is denser over its outer surface; innervation to the intergeniculate leaflet and ventral lateral geniculate nucleus is much less common. ${ }^{16}$ The suprachiasmatic nucleus is never innervated even when a graft is implanted immediately adjacent to it. The pattern of innervation is not entirely normal, however, even to regions such as the superior colliculus. Axons grow through it in an anomalous orientation, the innervation is more highly focussed near the surface, there is no evidence of segregation of inputs from host eye and transplant and, most important, the projection from the transplant to the colliculus is not topographically organised. ${ }^{17}$ The same specificity of innervation is seen even after implantation to anophthalmic mutants in which optic axons never leave the eye, ${ }^{18}$ suggesting that the cues used by transplant axons to reach their targets are not provided simply by previously established host projections.

The pattern of projections from grafts placed in mature animals has been studied in much less detail. It is clear that they do innervate host visual centres such as the superior colliculus and pretectum, but outgrowth is more local and limited. $^{16,19}$

\section{RELAY OF INFORMATION}

The anatomical studies indicate that the grafts do form specific projections to visual centres of the host brain but this is no guarantee that sensible information can be transmitted by way of the graft. It is quite possible that the information-processing capabilities of the graft itself could be defective; it is also likely that the transplantderived axons may not relay the same information as normal optic axons and furthermore that this information may not be properly relayed to the host neurons, because synapses may be differently disposed on cells of the host brain. Since there is no anatomical evidence for topographic order in the graft-host interconnections, behavioural studies have focussed in the first instance on luminance-driven responses.

The initial experiments examined gross potential responses in the transplant itself, and showed evidence of intensity-dependent light-driven activity. ${ }^{20.21}$ Unit activity recorded from the superior colliculus in response to illumination of the transplant showed discrete responses to light on, light off and to background intensity levels. Some units were profoundly inhibited by illumination. ${ }^{20.21}$ These findings compared closely with previous observations on normal rodent colliculus. ${ }^{22}$ The activation of host neurons to transplant illumination is not restricted to the primary nuclei such as the superior colliculus: specific responses can also be recorded from the cortical area 18a through a circuit that most probably involves the superior colliculus and nucleus lateralis posterior as intermediate relays. ${ }^{23}$ further work examining c-fos activation after transplant stimulation has shown not only that cells in primary nuclei are activated to produce electrical events but also that immediate-early genes are activated. ${ }^{24}$ While these results do show that the transplant is capable of mediating luminance-driven information to the host brain, they do not address the question of whether it can be used by the host animal to elicit appropriate behavioural responses. This was explored in a further set of studies.

\section{BEHAVIOURAL ACTIVATION}

In order to examine whether the host brain could use information delivered by way of the transplant to effect a range of behavioural strategies, a series of different tests were devised. The first examined the capability of transplants to mediate a pupilloconstrictor response in the host eye. This capitalised on the fact that under normal circumstances, if one eye is illuminated, pupilloconstriction results not only in the eye being stimulated but also in the other eye. We reasoned that if we illuminated a transplant, a similar pupilloconstrictor response of the host eye might be expected. The first studies ${ }^{19}$ were performed on rats with one eye removed at birth to produce heavier innervation of the host brain by the transplant axons, and with the remaining optic nerve cut prior to stimulation to rule out potential contamination by light scatter to the host eye. Under these conditions, illumination of the transplant caused pupilloconstriction of the host pupil. The amplitude of constriction varied among animals and this correlated with the density of innervation of the olivary pretectal nucleus, the area of the pretectum responsible for 
mediating pupilloconstriction. ${ }^{25}$ For any single animal, the response was highly predictable both within a single testing session and between testing sessions: it was intensity dependent, like the normal pupilloconstrictor response, but the threshold response was generally about $2 \log$ units higher than normal. Subsequent study showed that it could be elicited even when both eyes were maintained intact, and in these cases both gave a constrictor response to the transplant stimulation, although the amplitude of the response was not always the same for the two eyes. A series of control studies showed that contrary to expectations, there was no evidence of light scatter to the host eye.

This preparation has permitted careful quantification of transplant responsiveness, allowing us to identify factors that might help or hinder the effectiveness of transplant activation of the host pupilloconstrictor pathways. the basic biology of the response has received remarkably little attention given its value as a diagnostic tool. ${ }^{26}$ One study has suggested that it may be driven primarily by rodactivated pathways; ${ }^{26}$ other work has indicated that the response may be driven by two separate channels,

\section{Phase 1: Acquisition}
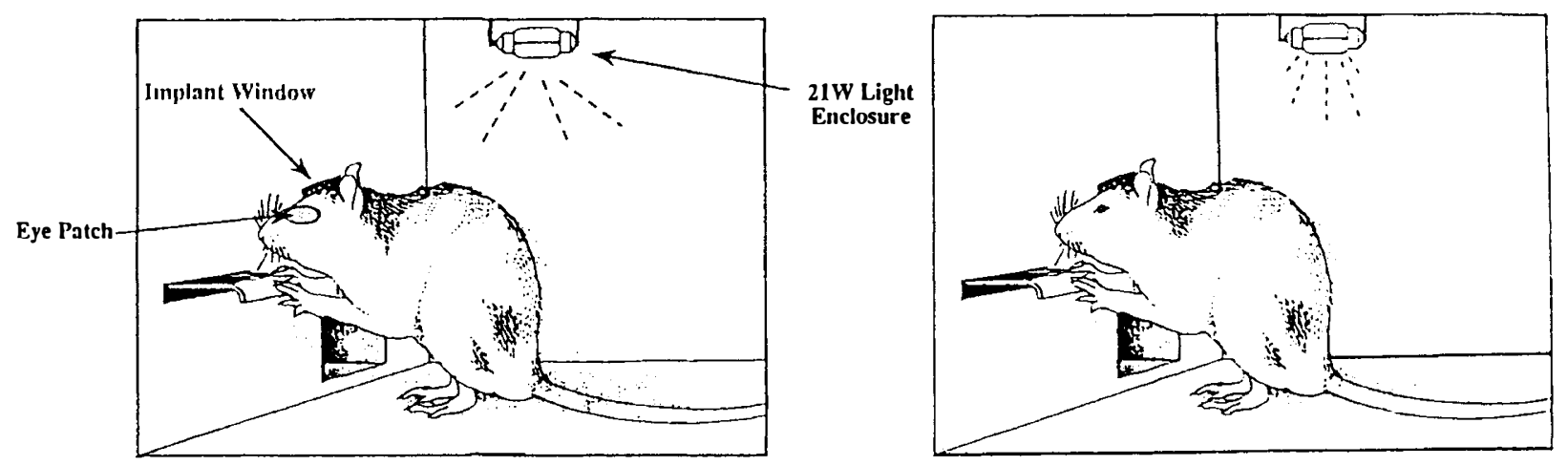

Experimental Group 1 (Retinal implant first)
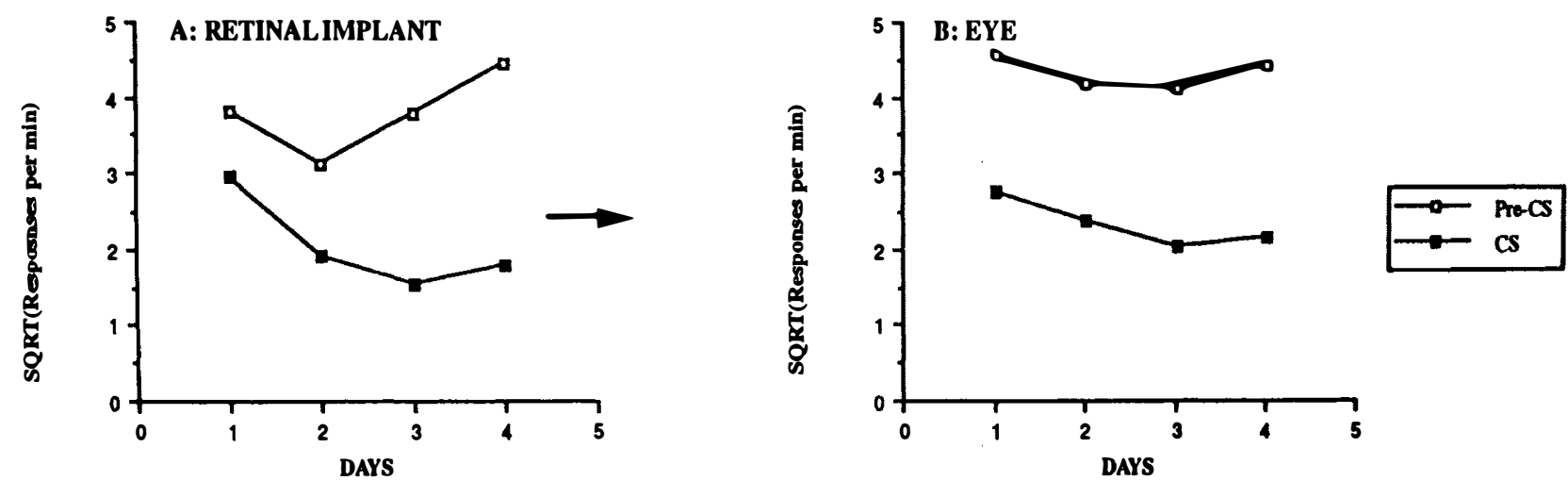

Fig. 1. Animal's lever-pressing rates for food reward over days before the onset of a light stimulus (Pre-CS) and during the presentation of a light stimulus (CS) which predicted the delivery of mild footshock. In phase 1 the light stimulus was presented to the transplant only, with an opaque eye patch placed over the animal's own eye (graph A). This was immediately followed by phase 2 where the light stimulus was presented to the animal's own eye with an opaque patch placed over the retinal implant (graph B). 
Phase 1: Acquisition

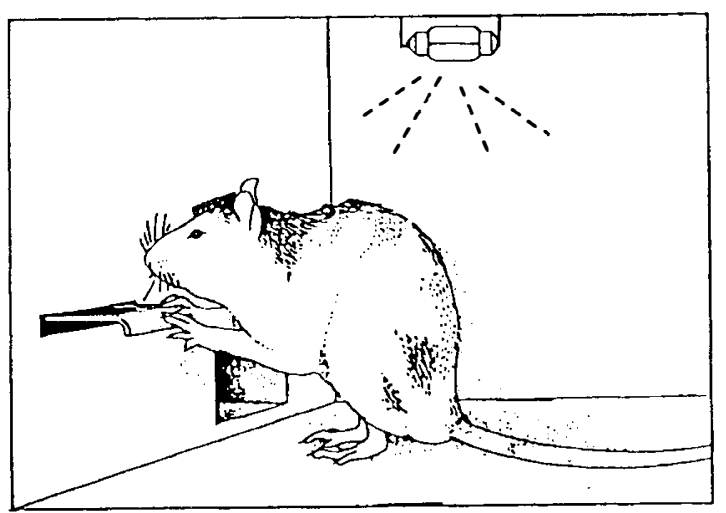

Phase 2: Transference

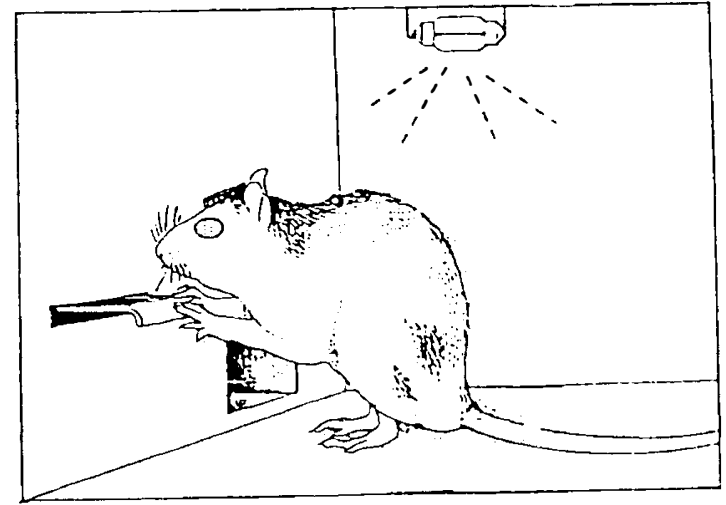

\section{Experimental Group 2 (Eye first)}
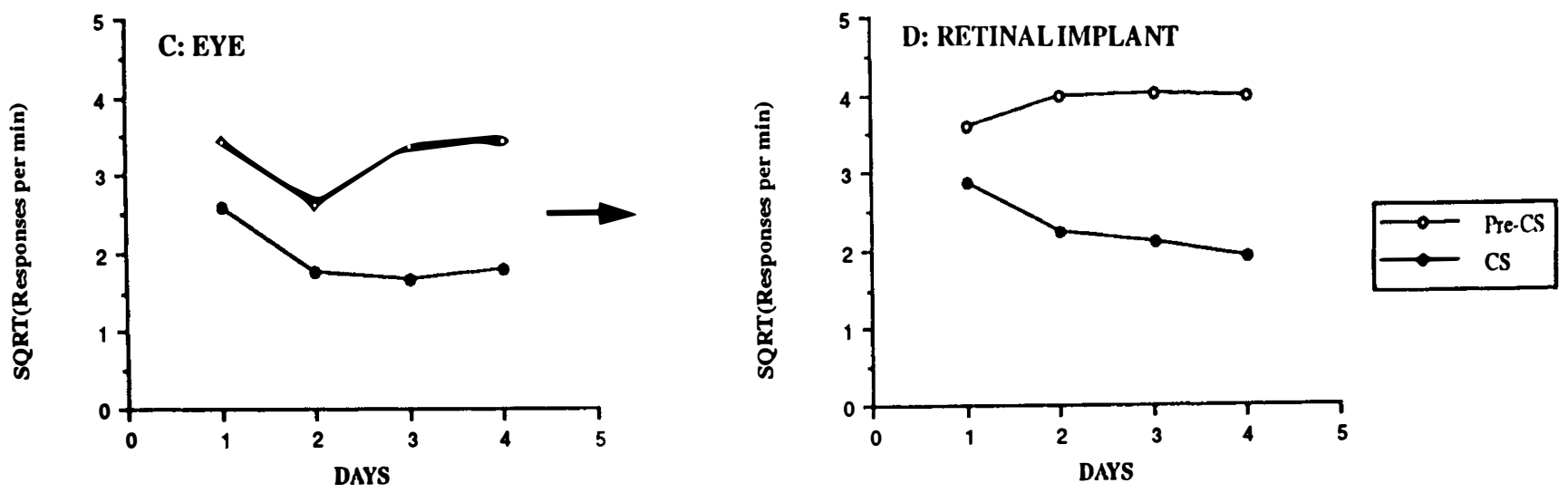

Fig. 2. Animal's lever-pressing rates for food reward over days before the onset of a light stimulus (Pre-CS) and during the presentation of a light stimulus $(C S)$ which predicted the delivery of mild footshock. In phase 1 the light stimulus was presented to the animal's own eye, with an opaque patch placed over the retinal implant (graph C). This was immediately followed by phase 2 where the light stimulus was presented to the transplant only with an opaque eye patch placed over the animal's own eye (graph D).

suppression. Rats with retinal implants were taught to press a lever for a food reward. Then, during the conditioning phase of the experiment, two periods were presented in which a tone was turned on for 5 minutes. Over the two 5 minute periods there were a total of eight pseudo-random presentations of a light for 15 seconds at the end of which a mild footshock was delivered. The baseline lever-pressing rate was recorded before the onset of any stimuli; during the tone 15 seconds prior to the onset of the light; and during the 15 second light onset. In separate groups of animals the light was presented either to the animal's own eye or, using a light guide, to the implanted retina. If animals were able to detect the light when presented to their retinal implant, their lever-pressing rate to retinal implant illumination would be lower than their lever-pressing rate either during tone onset or under baseline conditions. However, if the animals could not detect retinal implant illumination, then tone and light response rates would be similar to each other, approximating baseline response levels. The results showed that the animals were able to learn the response as well through the transplant as through the host eye. ${ }^{30}$ Subsequent work has shown that flashing light is a more salient stimulus than continuous illumination and that animals can also learn to respond to light offset as well as to illumination (Coffey et al., unpublished observations).

These observations showed that an animal can detect implant illumination, but they did not address the question of whether implant illumination was experientially equivalent to illuminating the normal eye. Our first attempt to address this issue capitalised on a well-established unconditioned photophobic response exhibited by rats. When placed in a brightly lit, novel environment, rats typically take cover in any available darkened area. ${ }^{33}$ If retinal implant illumination is experientially similar to conventionally detected light, a similar behaviour would 
be expected in animals with an exposed graft. We examined this using a large, open test arena with segments that were either brightly lit or covered. When viewing the arena using their normal eye, rats showed a characteristic photophobic response, spending significantly more time in the darkened segment of the open field. When they were first tested using their transplant, they appeared blind, spending equal amounts of time in the brightly lit and darkened areas. This suggested in the first analysis that implant illumination was not experientially similar to illumination of the animal's own eye. However, animals that were taught a light-shock association that depended on illumination of their retinal implant, subsequently exhibited photophobia. ${ }^{32}$ Why is this? Is it because the animal has learnt that light, whatever it may be perceived as, when presented to a retinal implant is treated as an aversive stimulus? Alternatively, until forced by the conditioning studies to attend to the transplant-mediated signal, they may not have been able to attach significance to this input. Recent findings (Coffey and Lund, unpublished observations) support the latter possibility: they are not merely learning an aversive association, but have to be taught about transplant illumination before they become 'aware' or possibly 'conscious' of it. The mechanisms underlying this are obviously of great interest.

Given that there is a degree of equivalence to stimuli relayed to the brain by either the transplant or the host eye, the further question arises as to whether there is transfer of learning from one to the other. In this set of experiments we found evidence to suggest that information could indeed be transferred from the retinal implant to the animal's host eye, but not apparently in the opposite direction from the animal's host eye to a retinal implant (Figs. 1, 2). One possible explanation for this asymmetry is that implanted retinae placed over the dorsal brainstem innervate only a subset of the normal visual centres. ${ }^{15}$

These observations show that information relayed by way of the transplant can modify behaviour patterns in the host animal. With the exception of the pupilloconstrictor response, the pathways whereby this occurs are not presently evident. Furthermore, it is not at all clear exactly what parameters of the visual input are used by the animal to trigger the behaviour: whether the animal actually 'sees' the stimulus or whether the response is an unconscious response more akin to blindsight. ${ }^{34}$ What is clear from the studies showing an interrelation between photophobic behaviour and conditioned suppression testing is that for certain functions it is not sufficient simply to have a connection between the transplant and the host brain for the animal to take advantage of the pathway, but it must be reinforced by suitable training regimens.

\section{CONCLUSION}

The work shows that it is possible to reconstruct circuits in both developing and mature brains that are capable of transmitting, in a logical fashion, certain aspects of sensory signals sufficient to effect appropriate patterns of response. It is clear that the connections are not absolutely normal but it is not evident at this point how much internal regulation of host connections occurs to compensate for abnormalities in the transplant input. It would be surprising, however, if topographic information could be extracted in the absence of a proper map, although it is possible, for example, that scaling of signal levels to produce appropriate responses to lights of a particular luminance could occur.

What relevance does this work have for addressing the potential use of transplantation strategies as a cure for blindness? Clearly it does show that a remarkable degree of reconstruction of functional circuits is possible, and it provides a suitable preparation in which to examine how to optimise strategies for improving not only the efficiency of the connections between transplant and host but also the development of the internal circuitry within the transplant itself. It is clear that axons from transplants placed in the adult brain fail to extend the long distance from the orbit to the visual centres of the brainstem, so that were it ever possible to replace a damaged eye with a transplanted retina, it would be necessary to combine it with a further transplant, such as a peripheral nerve graft, ${ }^{35}$ to serve as a conduit to promote long-distance fibre growth.

The work described here was supported by Action Research (UK), MRC, NIH and the Royal Society.

Key words: Connections, Retina, Transplantation, Visual responses.

\section{REFERENCES}

1. Bjorklund A, Gage FH. Neural grafting in animal-models of neurodegenerative diseases. Ann NY Acad Sci 1985: 457:53-81.

2. Gage FH. Intracerebral grafting of genetically modified cells acting as biological pumps. Trends Pharmacol Sci 1990;1 1:437-9.

3, Lund RD, Hauschka SD. Transplanted neural tissue develops connections with host brain. Science 1976; 193:582-4.

4. Sotelo C, Alvarado-Mallart RM. The reconstruction of cerebellar circuits. Trends Neurosci 1991;14:350-4.

5. Lund RD, Radel JD, Hankin MH, Yee KT, Banerjee R, Coffey PJ, Horsburgh GM. Intracerebral retinal transplants. In: DMK Lam, GM Bray, editors. Regeneration and plasticity in the visual system: proceedings of the retina research symposium. Cambridge, Mass.: MIT Press, 1992:125-46.

6. Radel JD, Das S, Lund RD. Development of light-activated pupilloconstriction in rats as mediated by normal and transplanted retinae. Eur J Neurosci 1992;4:603-15.

7. Perry VH, Lund RD, McLoon SC. Ganglion cells in retinae transplanted to newborn rats. J Comp Neurol 1985; 231:353-63.

8. McLoon S, Lund RD. Specific projections of retina transplanted to rat brain. Exp Brain Res 1980;40:273-82.

9. Lund RD, Rao K, Kunz HW, Gill TJ. Instability of neural xenografts placed in neonatal rat brains. Transplantation 1988;46:216-23.

10. Rao K, Lund RD, Kunz HW, Gill TJ. Immunological implications of xenogeneic and allogeneic transplantation to neonatal rats. Prog Brain Res 1988;78:281-6.

11. Lund RD, Rao K, Kunz HW, Gill TJ. Immunological considerations in neural transplantation. Transplant Proc 1989;21:3159-62. 
12. LaVail MM. Analysis of neurological mutants with inherited retinal degeneration. Invest Ophthal Vis Sci 1981; $21: 638-57$.

13. Banerjee R, Lund RD. A role for microglia in the maintenance of photoreceptors in retinal transplants lacking pigment-epithelium. J Neurocytol 1992;21:235-43.

14. Hankin MH, Lund RD. Directed early axonal outgrowth from retinal transplants into host rat brains. J Neurobiol 1990;21:1202-18.

15. Radel JD, Galli-Resta L, Lund RD. Plasticity in innervation of the rat superior colliculus by transplanted retinae as a result of eye removal at maturity. Exp Neurol 1991; 112:252-63.

16. Lund RD, Radel JD, Yee KT. Experimental manipulation of the developing rodent vidual system. In: SC Sharma, JW Fawcett, editors. Formation and regeneration of nerve connections, Boston, Mass.: Birkhäuser, 1993:72-90.

17. Galli L, Rao K, Lund RD. Transplanted rat retinae do not project in a topographic fashion on the host tectum. Exp Brain Res 1989;74:427-30.

18. Horsburgh GM, Lund RD, Hankin MH. Retinal transplants in congenitally blind mice: patterns of projection and synaptic connectivity. J Comp Neurol 1993;327:323-40.

19. Klassen H, Lund RD. Retinal transplants can drive a pupillary reflex in host rat brains. Proc Natl Acad Sci USA 1987;84:6958-60.

20. Simons DJ, Lund RD. Functional activity of rat brain-stem neurons regenerating axons along peripheral-nerve grafts. Dev Brain Res 1985;21:156-9.

21. Simons DJ, Lund RD. Fetal retinae transplanted over the tecta of neonatal rats respond to light and evoke patterned neuronal discharges in the host superior colliculus. Dev Brain Res 1985;21:156-9.

22. Fukuda Y, Iwama K. Visual receptive-field properties of single cells in the rat superior colliculus. Jpn J Physiol 1978;28:385-400.

23. Craner SL, Radel JD, Jen LS, Lund RD. Light-evoked cortical activity produced by illumination of intracranial retinal transplants: experimental studies in rats. Exp Neurol 1989;104:93-100.

24. Craner SL, Hoffman GE, Lund JS, Lund RD. C-fos labelling in the superior colliculus: activation by normal retinal pathways and pathways from intracranial retinal tranplants. Exp Neurol 1991;117:219-29.

25. Klassen H, Lund RD. Anatomical and behavioural correlates of a xenograft-mediated pupillary reflex. Exp Neurol 1988;102:102-8.

26. Birch EE, Birch DG. Pupillometric measures of retinal sensitivity in infants and adults with retinitis pigmentosa. Vision Res 1987;27:499-505.

27. Young RSL, Han B-C, Wu P-Y. Transient and sustained components of the pupillary responses evoked by luminance and colour. Vis Res 1993;33:437-46.

28. Trejo LJ, Cicerone CM. Retinal sensitivity measured by the pupillary light reflex in RCS and albino rats. Vis Res 1982;22:1163-71.

29. Whiteley SJO, Young MJ, Coffey PJ, Lund RD. Pupillary light reflex in RCS rats: age-related changes and comparisons with visual acuity. Soc Neurosci Abstr 1993;19:1418.

30. Coffey PJ, Lund RD, Rawlins JNP. Retinal transplantmediated learning in a conditioned suppression task in rats. Proc Natl Acad Sci USA 1989;86:7248-9.

31. Coffey PJ, Lund RD. Alerting and orienting responses mediated by intracerebral retinal transplants. Soc Neurosci Abstr 1991;17:557.

32. Coffey PJ, Lund RD, Rawlins JNP. Detecting the world through a retinal implant. Prog Brain Res 1990;82:269-75.

33. Odling-Smee FJ. The role of background stimuli during Pavlovian conditioning. QJ Exp Psychol 1975;27:201-9.

34. Cowey A, Stoerig P. The neurobiology of blindsight. Trends Neurosci 1991;14:140-5.

35. Vidal-Sanz M, Bray GM, Villegas-Perez MP, Thanos S, Aguayo AJ. Axonal regeneration and synapse formation in the superior colliculus by retinal ganglion-cells in the adult rat. J Neurosci 1987;7:2894-909. 